\title{
TRACTABILITY OF MULTIVARIATE INTEGRATION USING LOW-DISCREPANCY SEQUENCES
}

\author{
Shu TezukA \\ Dedicated to the memory of Professor Pierre Liardet
}

\begin{abstract}
We propose a notion of $(\mathbf{t}, \mathbf{e}, s)$-sequences in multiple bases, which unifies the Halton sequence and $(t, s)$-sequences under one roof, and obtain an upper bound of their discrepancy consisting only of the leading term. By using this upper bound, we improve the tractability results currently known for the Halton sequence, the Niederreiter sequence, the Sobol' sequence, and the generalized Faure sequence, and also give tractability results for the Xing-Niederreiter sequence and the Hofer-Niederreiter sequence, for which no results have been known so far.
\end{abstract}

\section{Communicated by Oto Strauch}

\section{Introduction}

Information based complexity (IBC) is one of the most exciting fields in theoretical computer science. Since the remarkable success of quasi-Monte Carlo methods for financial applications in the mid '90s (see, e.g., 8, 14, 19, 23, 24, 27]), tractability theory of multivariate problems, a frontier of IBC, quickly emerged and has been developing in many different directions in the last two decades [2, 13, 15, 28]. In particular, tractability of multivariate integration using lowdiscrepancy sequences, which form a mainstay of quasi-Monte Carlo methods, has been an important topic and many interesting results were already obtained (see, e.g., 3, 6, 16, 25, 26]).

The aim of this paper is to improve the currently known tractability results on low-discrepancy sequences. The key idea for the improvement is briefly explained by using the case of the Halton sequence. Hickernell and Wang [6]

2010 Mathematics Subject Classification: Primary 11K38; Secondary 11K06.

Keywords: discrepancy, low-discrepancy sequences, multivariate integration, tractability, $(t, e, s)$-sequences. 


\section{SHU TEZUKA}

proved the strong tractability with the exponent 1 for the Halton sequence under a condition, $\sum_{i=1}^{\infty} \gamma_{i}^{1 / 2} i \log i<\infty$, where $\gamma_{1} \geq \gamma_{2} \geq \cdots \geq 0$ are weights for the weighted Sobolev space of integrands. Their analysis is based on the upper bound by Niederreiter [11] for the star discrepancy $D_{s}^{*}\left(P_{N}\right)$ of the first $N$ points, denoted by $P_{N}$, of the $s$-dimensional Halton sequence, which is given as

$$
\begin{aligned}
D_{s}^{*}\left(P_{N}\right) & \leq \frac{1}{N}\left(s+\prod_{i=1}^{s}\left(\frac{\left(p_{i}-1\right) \log N}{2 \log p_{i}}+\frac{p_{i}+1}{2}\right)\right) \\
& =\prod_{i=1}^{s}\left(\frac{p_{i}-1}{2 \log p_{i}}\right) \frac{(\log N)^{s}}{N}+O\left(\frac{(\log N)^{s-1}}{N}\right)
\end{aligned}
$$

for all $N>1$, where $p_{i}$ is the $i$ th smallest prime for $i=1,2, \ldots$ On the other hand, what happens if we use the upper bound by Halton [5] published more than half a century ago, which is given as

$$
D_{s}^{*}\left(P_{N}\right) \leq \prod_{i=1}^{s}\left(\frac{3 p_{i}-2}{\log p_{i}}\right) \frac{(\log N)^{s}}{N}
$$

for all $N>1$ ? We should notice that Halton's bound has only the leading term without any additional terms, while the leading constant is bigger than that of Niederreiter. According to the prime number theorem, $p_{i}=O(i \log i)$, the right-hand side of (11) is further bounded by $N^{-1} \prod_{i=1}^{s}(C i \log N)$, where $C$ is a positive constant. In fact, this bound eventually leads us to a weaker condition, $\sum_{i=1}^{\infty} \gamma_{i}^{1 / 2} i<\infty$, under which the strong tractability with the exponent 1 holds.

The organization of this paper is as follows. In Section 2, we introduce a general concept of $(\mathbf{t}, \mathbf{e}, s)$-sequences in multiple bases, which unifies the Halton sequence and $(t, s)$-sequences under one roof, and give an upper bound consisting only of the leading term like (11) for the discrepancy of their first $N(>1)$ points. This result yields upper bounds for the discrepancies of the Halton sequence, of the Niederreiter sequence, of the Sobol' sequence, of the generalized Faure sequence, of the Xing-Niederreiter sequence, and of the Hofer-Niederreiter sequence. In Section 3, based on the discrepancy upper bounds obtained in Section 2, we analyze the tractability for finite-order weights using points from the Halton sequence, the Niederreiter sequence, the Sobol' sequence, and the generalized Faure sequence, and improve the exising results. Then, we analyze the tractability using points from the Xing-Niederreiter sequence and the HoferNiederreiter sequence, for which no results have been known so far, and present new results for finite-order weights. In the final section, we discuss the tractability using points from the Niederreiter-Xing sequence. 


\section{2. $(\mathbf{t}, \mathbf{e}, s)$-sequences in multiple bases}

First, we introduce the definition of discrepancy [2, 10, 11]. For a point set $P_{N}=\left\{X_{0}, X_{1}, \ldots, X_{N-1}\right\}$ of $N$ points in $[0,1]^{s}$ and an interval $J \subseteq[0,1]^{s}$, we define $A_{N}(J)$ as the number of $n, 0 \leq n \leq N-1$, with $X_{n} \in J$ and $\mu(J)$ is the volume of $J$. Then the star discrepancy of $P_{N}$ is defined by

$$
D_{s}^{*}\left(P_{N}\right)=\sup _{J}\left|\frac{A_{N}(J)}{N}-\mu(J)\right|,
$$

where the supremum is taken over all intervals $J$ of the form $\prod_{i=1}^{s}\left[0, \alpha_{i}\right)$ for $0 \leq \alpha_{i} \leq 1$.

Let $\mathbf{b}=\left(b_{1}, \ldots, b_{s}\right)$ be an integer vector with $b_{i} \geq 2(1 \leq i \leq s)$. An elementary interval in multiple bases $\left(b_{1}, \ldots, b_{s}\right)$ is an interval of the form

$$
E_{\mathbf{b}}\left(j_{1}, \ldots, j_{s}\right)=\prod_{i=1}^{s}\left[\frac{a_{i}}{b_{i}^{j_{i}}}, \frac{a_{i}+1}{b_{i}^{j_{i}}}\right), \quad\left(0 \leq a_{i}<b_{i}^{j_{i}}, j_{i} \geq 0\right),
$$

where $a_{i}$ and $j_{i}$ are integers for $i=1, \ldots, s$.

Definition 1. Let $\mathbf{t}=\left(t_{1}, \ldots, t_{s}\right), \mathbf{m}=\left(m_{1}, \ldots, m_{s}\right), \mathbf{e}=\left(e_{1}, \ldots, e_{s}\right)$ and $\mathbf{j}=\left(j_{1}, \ldots, j_{s}\right)$ be integer vectors, where $t_{i} \geq 0, m_{i} \geq 0, e_{i} \geq 1$ and $j_{i} \geq 0$ for $i=1, \ldots, s$. Let $\mathbf{t}$ and $\mathbf{m}$ be vectors with $t_{i} \leq m_{i}(1 \leq i \leq s)$ such that $\mathbf{m}-\mathbf{t} \in M(\mathbf{e}):=\left\{\left(e_{1} j_{1}, \ldots, e_{s} j_{s}\right) \mid j_{i} \geq 0(1 \leq i \leq s)\right\}$. Denote $\mathbf{b}^{\mathbf{m}}=\prod_{i=1}^{s} b_{i}^{m_{i}}$. A $(\mathbf{t}, \mathbf{m}, \mathbf{e}, s)$-net in multiple bases $\left(b_{1}, \ldots, b_{s}\right)$ is a point set of $\mathbf{b}^{\mathbf{m}}$ points such that $A_{\mathbf{b}^{\mathrm{m}}}(E)=\prod_{i=1}^{s} b_{i}^{t_{i}}$ for every elementary interval $E=$ $E_{\mathbf{b}}\left(e_{1} j_{1}, \ldots, e_{s} j_{s}\right)$ in multiple bases $\left(b_{1}, \ldots, b_{s}\right)$ with $\mu(E)=\prod_{i=1}^{s} b_{i}^{t_{i}-m_{i}}$ and $\mathbf{j}$ satisfying $\left(e_{1} j_{1}, \ldots, e_{s} j_{s}\right)=\mathbf{m}-\mathbf{t}$.

Definition 2. A $(\mathbf{t}, \mathbf{e}, s)$-sequence in multiple bases $\left(b_{1}, \ldots, b_{s}\right)$ is an infinite sequence, $X=\left(X_{n}\right)_{n \geq 0}$, of points in $[0,1]^{s}$ such that for all integers $\ell \geq 0$ and all $\mathbf{m}$ with $m_{i} \geq t_{i}(1 \leq i \leq s)$ satisfying $\mathbf{m}-\mathbf{t} \in M(\mathbf{e})$, the point set $\left\{\left[X_{\ell \mathbf{b}^{\mathbf{m}}}\right]_{\mathbf{b}, \mathbf{m}}, \ldots,\left[X_{(\ell+1) \mathbf{b}^{\mathbf{m}}-1}\right]_{\mathbf{b}, \mathbf{m}}\right\}$ is a $(\mathbf{t}, \mathbf{m}, \mathbf{e}, s)$-net, where $\left[X_{n}\right]_{\mathbf{b}, \mathbf{m}}$ means the $m_{i}$-digit truncation in base $b_{i}$ of the $i$ th coordinate of a point $X_{n}$ for $1 \leq i \leq s$.

It is obvious that a $(t, \mathbf{e}, s)$-sequence in a single base $b[20$ is identical to a $(\mathbf{t}, \mathbf{e}, s)$-sequence in multiple bases $\left(b_{1}, \ldots, b_{s}\right)$, where $b_{1}=\cdots=b_{s}=b$ and $\mathbf{t}=\left(t_{1}, \ldots, t_{s}\right)$ is any tuple satisfying $t=t_{1}+\cdots+t_{s}$.

REMARK 1. The $s$-dimensional Halton sequence [5] is a $(\mathbf{t}, \mathbf{e}, s)$-sequence in multiple bases $\left(b_{1}, \ldots, b_{s}\right)$ such that $\mathbf{t}=(0, \ldots, 0), \mathbf{e}=(1, \ldots, 1)$, and $b_{i}$ is the $i$ th smallest prime for $i=1, \ldots, s$. 


\section{SHU TEZUKA}

REMARK 2. The $s$-dimensional Niederreiter sequence in base $b[11$ is a $(\mathbf{t}, \mathbf{e}, s)$ -sequence in multiple bases $\left(b_{1}, \ldots, b_{s}\right)$ such that $\mathbf{t}=(0, \ldots, 0), \mathbf{e}=\left(e_{1}, \ldots, e_{s}\right)$, and $b_{1}=\cdots=b_{s}=b$, where $e_{i}, 1 \leq i \leq s$, is equal to the degree of the $i$ th polynomial from the list of all monic irreducible polynomials over $\mathrm{GF}(b)$ sorted in nondecreasing order of degree.

REMARK 3. The $s$-dimensional Sobol' sequence [17] is a (t, e, $s)$-sequence in multiple bases $\left(b_{1}, \ldots, b_{s}\right)$ such that $\mathbf{t}=(0, \ldots, 0), \mathbf{e}=\left(e_{1}, \ldots, e_{s}\right)$, and $b_{1}=\cdots=b_{s}=2$, where $e_{1}=1$, and $e_{i}, 2 \leq i \leq s$, is equal to the degree of the $(i-1)$-th polynomial from the list of all primitive polynomials over $\mathrm{GF}(2)$ sorted in nondecreasing order of degree.

REMARK 4. The $s$-dimensional generalized Faure sequence in base $b[18$ is a $(\mathbf{t}, \mathbf{e}, s)$-sequence in multiple bases $\left(b_{1}, \ldots, b_{s}\right)$ such that $\mathbf{t}=(0, \ldots, 0), \mathbf{e}=$ $(1, \ldots, 1)$, and $b_{1}=\cdots=b_{s}=b$, where $b$ is the smallest prime with $b \geq s$.

REMARK 5. Each of the $s$-dimensional Xing-Niederreiter sequence in base $b$ and genus $g$ [29] and the $s$-dimensional Hofer-Niederreiter sequence in base $b$ and genus $g$ [7] is a $(\mathbf{t}, \mathbf{e}, s)$-sequence in multiple bases $\left(b_{1}, \ldots, b_{s}\right)$ such that $\mathbf{t}=\left(t_{1}, \ldots, t_{s}\right)$ is any tuple satisfying $g=t_{1}+\cdots+t_{s}, \mathbf{e}=\left(e_{1}, \ldots, e_{s}\right)$, and $b_{1}=\cdots=b_{s}=b$, where $e_{i}$ is equal to the degree of the $i$ th place from the list of all places sorted in nondecreasing order of degree.

Next, we will give a theorem on the upper bound for the discrepancy of $(\mathbf{t}, \mathbf{e}, s)$-sequences in multiple bases $\left(b_{1}, \ldots, b_{s}\right)$. Before that, we need the following lemmas.

LEMMA 1. Let $\left(b_{1}, \ldots, b_{s}\right)$ be an integer vector with $b_{i} \geq 2(1 \leq i \leq s)$, and $\mathbf{e}=\left(e_{1}, \ldots, e_{s}\right)$ and $\mathbf{j}=\left(j_{1}, \ldots, j_{s}\right)$ be integer vectors with $e_{i} \geq 1$ and $j_{i} \geq 0$ for $i=1, \ldots, s$. Let $I$ be an interval given by

$$
I=\prod_{i=1}^{s}\left[\frac{a_{i}}{b_{i}^{e_{i} j_{i}}}, \frac{c_{i}}{b_{i}^{e_{i} j_{i}}}\right), \quad\left(0 \leq a_{i}<c_{i} \leq b_{i}^{e_{i} j_{i}}\right),
$$

where $a_{i}$ and $c_{i}$ are integers for $i=1, \ldots, s$. Denote $B=\prod_{i=1}^{s} b_{i}^{t_{i}}$ and $\mathbf{b}^{\mathbf{e j}}=\prod_{i=1}^{s} b_{i}^{e_{i} j_{i}}$. Then, for the first $N$ points of the truncated version of a $(\mathbf{t}, \mathbf{e}, s)$-sequence in multiple bases $\left(b_{1}, \ldots, b_{s}\right)$, we have

$$
\left|A_{N}(I)-N \mu(I)\right| \leq B \prod_{i=1}^{s}\left(c_{i}-a_{i}\right)
$$

for every positive integer $N$, and $A_{N}(I) \leq B \prod_{i=1}^{s}\left(c_{i}-a_{i}\right)$ if $N \leq B \mathbf{b}^{\mathbf{e j}}$, where the truncation size is taken to be large enough depending on $\mathbf{j}$. 
Proof. From Definition 2, for the truncated version of a $(\mathbf{t}, \mathbf{e}, s)$-sequence in multiple bases $\left(b_{1}, \ldots, b_{s}\right)$, we see that for any integer $\ell \geq 0$,

$$
A_{\ell B \mathbf{b}^{\mathrm{ej}}}(I)=\ell B \prod_{i=1}^{s}\left(c_{i}-a_{i}\right)
$$

The last statement follows by taking $\ell=1$ and the fact that $A_{N}(I)$ is nondecreasing in $N$. For an arbitrary $N$, there is $\ell$ such that $\ell B \mathbf{b}^{\mathbf{e j}} \leq N<(\ell+1) B \mathbf{b}^{\mathbf{e j}}$. Thus, we have

$$
A_{N}(I)-N \mu(I) \leq A_{(\ell+1) B \mathbf{b}^{\mathbf{e j}}}(I)-\ell B \mathbf{b}^{\mathbf{e j}} \prod_{i=1}^{s} \frac{c_{i}-a_{i}}{b_{i}^{e_{i} j_{i}}}=B \prod_{i=1}^{s}\left(c_{i}-a_{i}\right),
$$

and

$$
N \mu(I)-A_{N}(I) \leq(\ell+1) B \mathbf{b}^{\mathbf{e j}} \prod_{i=1}^{s} \frac{c_{i}-a_{i}}{b_{i}^{e_{i} j_{i}}}-A_{\ell B \mathbf{b}^{\mathbf{e j}}}(I)=B \prod_{i=1}^{s}\left(c_{i}-a_{i}\right) .
$$

This completes the proof.

Lemma 2. Let $b \geq 4$ be an integer. Then, we have $x^{b} \geq b^{x}$ for $2 \leq x \leq b$.

Pr o of. Since $\log x$ is a concave function, if $b \geq 4$ we have

$$
\frac{x}{b} \leq \frac{\log x}{\log b}
$$

for $2 \leq x \leq b$. Thus, the proof is complete.

Lemma 3. Let $b_{1}, \ldots, b_{s}$ be integers with $2 \leq b_{1} \leq \cdots \leq b_{s}$. Then for any integer $N$ with $1<N \leq b_{s}$ we have

$$
\prod_{i=1}^{s}\left(\left(2 b_{i}-1\right) \log _{b_{i}} N\right)>N
$$

Proof. By Lemma 2 and simple calculations for $b_{s}=2$ and 3, we have

$$
N^{2 b_{s}-1}>b_{s}^{N}
$$

for $b_{s} \geq 2$ and $2 \leq N \leq b_{s}$. Therefore, $\left(2 b_{s}-1\right) \log _{b_{s}} N>N \log _{b_{s}} b_{s}=N$. For $i=1, \ldots, s-1$, we observe $\left(2 b_{i}-1\right) \log _{b_{i}} N \geq\left(2 b_{i}-1\right) \log _{b_{i}} 2>\log _{b_{i}} b_{i}=1$. Thus, the proof is complete.

We now prove the main theorem in this section, whose proof is based on an excellent exposition by Matoušek [10, pp. 41-43]. 
TheOREM 1. Let $\left(b_{1}, \ldots, b_{s}\right)$ be an integer vector with $b_{i} \geq 2(1 \leq i \leq s)$. The star discrepancy for the first $N$ points, denoted by $P_{N}$, of a $(\mathbf{t}, \mathbf{e}, s)$-sequence in multiple bases $\left(b_{1}, \ldots, b_{s}\right)$ satisfies

$$
N D_{s}^{*}\left(P_{N}\right) \leq B \prod_{i=1}^{s}\left(\frac{2 b_{i}^{e_{i}}-1}{e_{i} \log b_{i}}\right)(\log N)^{s}
$$

for all $N>1$, where $B=\prod_{i=1}^{s} b_{i}^{t_{i}}$.

Pr o of. Take any $\mathbf{z}=\left(z^{(1)}, \ldots, z^{(s)}\right) \in[0,1]^{s}$. Each $z^{(i)}$ is expanded in base $b_{i}^{e_{i}}$ as $\sum_{j=1}^{\infty} a_{j}^{(i)} b_{i}^{-e_{i} j}$, with $0 \leq a_{j}^{(i)} \leq b_{i}^{e_{i}}-1$. Let $n_{i}=\left\lceil\log _{b_{i}^{e_{i}}} N\right\rceil$ and define $z_{0}^{(i)}=0$ and $z_{n_{i}+1}^{(i)}=z^{(i)}$. Consider the numbers $z_{k}^{(i)}=\sum_{j=1}^{k} a_{j}^{(i)} b_{i}^{-e_{i} j}$ for $k=1, \ldots, n_{i}$. Then, we obtain the splitting of $J=\prod_{i=1}^{s}\left[0, z^{(i)}\right)$ as

$$
I(\mathbf{j})=\prod_{i=1}^{s}\left[z_{j_{i}}^{(i)}, z_{j_{i}+1}^{(i)}\right)
$$

where $\mathbf{j}=\left(j_{1}, \ldots, j_{s}\right)$ with $0 \leq j_{i} \leq n_{i}(1 \leq i \leq s)$. For the truncated version of $(\mathbf{t}, \mathbf{e}, s)$-sequences in multiple bases, Lemma 1 gives

$$
\left|A_{N}(I(\mathbf{j}))-N \mu(I(\mathbf{j}))\right| \leq B \prod_{i=1}^{s}\left(b_{i}^{e_{i}}-1\right)
$$

for all $\mathbf{j}$ with $0 \leq j_{i}<n_{i}(1 \leq i \leq s)$. Denote the remaining intervals by

$$
R_{h}=\bigcup_{\mathbf{j} \in \mathcal{J}_{h}} I(\mathbf{j}),
$$

for $h=1, \ldots, s$, where $\mathcal{J}_{h}=\left\{\left(j_{1}, \ldots, j_{s}\right) \mid 0 \leq j_{i} \leq n_{i}(1 \leq i \neq h \leq s)\right.$ and $\left.j_{h}=n_{h}\right\}$. Since $\mu\left(R_{h}\right) \leq b_{h}^{-e_{h} n_{h}} \leq 1 / N$ and $R_{h}$ contains at most $B$ points, we have

$$
\left|A_{N}\left(R_{h}\right)-N \mu\left(R_{h}\right)\right| \leq B
$$

for $h=1, \ldots, s$. Therefore,

$$
\left|A_{N}(J)-N \mu(J)\right| \leq B \prod_{i=1}^{s}\left(b_{i}^{e_{i}}-1\right) \times \prod_{i=1}^{s}\left\lceil\log _{b_{i}^{e_{i}}} N\right\rceil+B s
$$

for any $J$. Since the right-hand side is independent of the interval $J$, it is an upper bound of $N D_{s}^{*}\left(P_{N}\right)=\sup _{J}\left|A_{N}(J)-N \mu(J)\right|$.

Assuming that $N \geq \max _{1 \leq i \leq s} b_{i}^{e_{i}}$, we can further bound the right-hand side of (3) from above as follows: 
TRACTABILITY OF MULTIVARIATE INTEGRATION

$$
\begin{aligned}
N D_{s}^{*}\left(P_{N}\right) & \leq B\left(\prod_{i=1}^{s}\left(\left(b_{i}^{e_{i}}-1\right)\left(\log _{b_{i}^{e_{i}}} N+1\right)\right)+s\right) \\
& \leq B\left(\prod_{i=1}^{s}\left(2\left(b_{i}^{e_{i}}-1\right) \log _{b_{i}^{e_{i}}} N\right)+s\right) \\
& \leq B \prod_{i=1}^{s}\left(\left(2 b_{i}^{e_{i}}-2\right) \log _{b_{i}^{e_{i}}} N+1\right) \\
& \leq B \prod_{i=1}^{s}\left(\left(2 b_{i}^{e_{i}}-1\right) \log _{b_{i}^{e_{i}}} N\right)
\end{aligned}
$$

In fact, this upper bound holds for all $N>1$, because, from Lemma 3, it is bigger than $N\left(\geq N D_{s}^{*}\left(P_{N}\right)\right)$ for all $N$ with $1<N \leq \max _{1 \leq i \leq s} b_{i}^{e_{i}}$.

Hence, the discrepancy bound (2) for the truncated version is obtained. As shown in 20], the discrepancy bound for the untruncated version remains the same as the truncated version. Thus, the proof is complete.

If the discrepancy is analyzed based on Atanassov's signed splitting method [1], we obtain

$$
N D_{s}^{*}\left(P_{N}\right) \leq \frac{B}{s !} \prod_{i=1}^{s}\left(\frac{b_{i}^{e_{i}}-1}{2 e_{i} \log b_{i}}\right)(\log N)^{s}+O\left((\log N)^{s-1}\right)
$$

for all $N>1$ [21]. (The proof is a simple generalization of the one given in [22] for $(t, \mathbf{e}, s)$-sequences in a single base $b$.) Although the leading constant becomes smaller than the one in Theorem 1, it looks difficult to apply this upper bound to the tractability analysis to obtain better results.

Based on the above theorem, we can derive the following corollaries for the four types of low-discrepancy sequences.

Corollary 1. Let $P_{N}$ be the first $N$ points of the s-dimensional Halton sequence. Let $P_{N}^{u}$ be the projection of $P_{N}$ on the lower dimensional space $[0,1]^{|u|}$. Then for any nonempty subset $u \subseteq\{1, \ldots, s\}$, the star discrepancy of $P_{N}^{u}$ satisfies

$$
D_{|u|}^{*}\left(P_{N}^{u}\right) \leq \frac{1}{N} \prod_{i \in u}(C i \log N)
$$

for all $N>1$, where $C=6$.

P r o o f. From Remark 1 and Theorem 1], we have

$$
N D_{|u|}^{*}\left(P_{N}^{u}\right) \leq \prod_{i \in u}\left(\frac{2 b_{i}-1}{\log b_{i}} \log N\right)
$$

for all $N>1$, where $b_{i}$ is the $i$ th smallest prime for $i=1, \ldots, s$. 
Since $b_{i} / \log b_{i}<3 i$ for $i=1,2, \ldots$ (see, e.g., Dusart [4]), the right-hand side of (4) is further bounded from above by

$$
\prod_{i \in u}(6 i \log N) .
$$

Thus, the proof is complete.

Corollary 2. Let $P_{N}$ be the first $N$ points of the s-dimensional Niederreiter sequence in base b. Let $P_{N}^{u}$ be the projection of $P_{N}$ on the lower dimensional space $[0,1]^{|u|}$. Then for any nonempty subset $u \subseteq\{1, \ldots, s\}$, the star discrepancy of $P_{N}^{u}$ satisfies

$$
D_{|u|}^{*}\left(P_{N}^{u}\right) \leq \frac{1}{N} \prod_{i \in u}(C i \log N)
$$

for all $N>1$, where $C=4 b^{2} / \log b$.

Pr o of. From Remark 2 and Theorem 1, we have

$$
N D_{|u|}^{*}\left(P_{N}^{u}\right) \leq \prod_{i \in u}\left(\frac{2 b^{e_{i}}-1}{e_{i} \log b} \log N\right)
$$

for all $N>1$, where $e_{i}, 1 \leq i \leq s$, is equal to the degree of the $i$ th polynomial from the list of all monic irreducible polynomials over $\mathrm{GF}(b)$ sorted in nondecreasing order of degree.

According to Niederreiter [11], we have

$$
e_{i} \leq \log _{b} i+\log _{b} \log _{b}(i+b)+2 \text {. }
$$

Thus, the right-hand side of (5) is bounded from above by

$$
\prod_{i \in u}\left(\frac{2 b^{2} i \log _{b}(i+b)}{e_{i} \log b} \log N\right) .
$$

Since $\log _{b}(i+b) \leq 2 e_{i}$ for $i=1,2, \ldots$, we can further bound it by

$$
\prod_{i \in u}\left(\frac{4 b^{2} i}{\log b} \log N\right) \text {. }
$$

Thus, the proof is complete.

Corollary 3. Let $P_{N}$ be the first $N$ points of the s-dimensional Sobol' sequence. Let $P_{N}^{u}$ be the projection of $P_{N}$ on the lower dimensional space $[0,1]^{|u|}$. Then for any nonempty subset $u \subseteq\{1, \ldots, s\}$, the star discrepancy of $P_{N}^{u}$ satisfies

$$
D_{|u|}^{*}\left(P_{N}^{u}\right) \leq \frac{1}{N} \prod_{i \in u}\left(C i \log _{2} \log _{2}(i+3) \log N\right)
$$

for all $N>1$, where $C>1$ is a constant. 
Pr o of. From Remark 3 and Theorem 1, we have

$$
N D_{|u|}^{*}\left(P_{N}^{u}\right) \leq \prod_{i \in u}\left(\frac{2^{e_{i}+1}-1}{e_{i} \log 2} \log N\right)
$$

for all $N>1$, where $e_{1}=1$, and $e_{i}, 2 \leq i \leq s$, is equal to the degree of the $(i-1)$ th polynomial from the list of all primitive polynomials over $\mathrm{GF}(2)$ sorted in nondecreasing order of degree.

According to Sobol' [17, we have

$$
e_{i} \leq \log _{2} i+\log _{2} \log _{2}(i+1)+\log _{2} \log _{2} \log _{2}(i+3)+c
$$

for $i=1,2, \ldots$, where $c$ is a positive constant independent of $i$.

Thus, the right-hand side of (6) is bounded from above by

$$
\prod_{i \in u}\left(\frac{2^{c+1} i \log _{2}(i+1) \log _{2} \log _{2}(i+3)}{e_{i} \log 2} \log N\right) .
$$

Since $\log _{2}(i+1)<2 e_{i}$ for $i=1,2, \ldots$, we can further bound it by

$$
\prod_{i \in u}\left(\frac{2^{c+2}}{\log 2} i \log _{2} \log _{2}(i+3) \log N\right) \text {. }
$$

Thus, the proof is complete.

CoRollary 4. Let $P_{N}$ be the first $N$ points of the s-dimensional generalized Faure sequence in base b. Let $P_{N}^{u}$ be the projection of $P_{N}$ on the lower dimensional space $[0,1]^{|u|}$. Then for any nonempty subset $u \subseteq\{1, \ldots, s\}$, the star discrepancy of $P_{N}^{u}$ satisfies

$$
D_{|u|}^{*}\left(P_{N}^{u}\right) \leq \frac{1}{N}\left(C \frac{s \log N}{\log s}\right)^{|u|}
$$

for all $N>1$ and $s>1$, where $C=4$.

Pr o of. From Remark 4 and Theorem 1, we have

$$
N D_{|u|}^{*}\left(P_{N}^{u}\right) \leq\left(\frac{2 b-1}{\log b} \log N\right)^{|u|}
$$

for all $N>1$, where $b$ is the smallest prime with $b \geq s$.

According to the Bertrand postulate, $s \leq b \leq 2 s$, the right-hand side of (17) is bounded from above by

$$
\left(\frac{4 s \log N}{\log s}\right)^{|u|} \text {. }
$$

Thus, the proof is complete. 
Corollary 5. Let $P_{N}$ be the first $N$ points of the s-dimensional Xing-Niederreiter sequence in base $b$ and genus $g$ or of the $s$-dimensional Hofer-Niederreiter sequence in base $b$ and genus $g$. Let $P_{N}^{u}$ be the projection of $P_{N}$ on the lower dimensional space $[0,1]^{|u|}$. Then for any nonempty subset $u \subseteq\{1, \ldots, s\}$, the star discrepancy of $P_{N}^{u}$ satisfies

$$
D_{|u|}^{*}\left(P_{N}^{u}\right) \leq \frac{b^{g}}{N} \prod_{i \in u}(C i \log N)
$$

for all $N>1$, where $C>1$ is a constant.

Pr o of. From Remark 5 and Theorem [1, we have

$$
N D_{|u|}^{*}\left(P_{N}^{u}\right) \leq b^{g} \prod_{i \in u}\left(\frac{2 b^{e_{i}}-1}{e_{i} \log b} \log N\right)
$$

for all $N>1$, where $e_{i}, 1 \leq i \leq s$, is equal to the degree of the $i$ th place from the list of all places sorted in nondecreasing order of degree. According to the prime number theorem for global function fields [9]:

$$
\#(e)=\frac{b}{b-1} \cdot \frac{b^{e}}{e}+o\left(\frac{b^{e}}{e}\right) \quad \text { as } \quad e \rightarrow \infty,
$$

where $\#(e), e=1,2, \ldots$, is the number of places of degree $\leq e$, we can further bound (8) by

$$
b^{g} \prod_{i \in u}(C i \log N),
$$

because $\#\left(e_{i}-1\right)<i \leq \#\left(e_{i}\right)$ for $i=1,2, \ldots$ Thus, the proof is complete. Notice that the theorem (9) holds independently of the genus $g$.

\section{Tractability analysis of several low-discrepancy sequences}

In this section, we apply the results obtained in the preceding section to the tractability analysis for finite-order weights, of the Halton sequence, of the Niederreiter sequence, of the Sobol' sequence, of the generalized Faure sequence, of the Xing-Niederreiter sequence, and of thel Hofer-Niederreiter sequence. First, we define the worst case integration error by

$$
e\left(P_{N} ; H\right)=\sup _{\|f\|_{H} \leq 1}\left|\int_{[0,1]^{s}} f(\mathbf{x}) d \mathbf{x}-\frac{1}{N} \sum_{i=0}^{N-1} f\left(X_{i}\right)\right|
$$


where $P_{N}=\left\{X_{0}, X_{1}, \ldots, X_{N-1}\right\}$ is a set of $N$ points in $[0,1]^{s}$ and $H$ denotes a normed space of integrands $f(\mathbf{x})$. We denote the initial error, which indicates the error when $P_{N}$ is empty, by $e(0 ; H)$. In this section, we consider two types of spaces. One is the anchored Sobolev space with an arbitrary anchor $\mathbf{a}=\left(a_{1}, \ldots, a_{s}\right) \in[0,1]^{s}$, and the other is the unanchored Sobolev space. For more detailed background and theoretical properties of these spaces, consult the blue book by Novak and Woźniakowski [13. As in [13], we say that the weights $\gamma=\left\{\gamma_{s, u}\right\}$ are finite-order if there exists an integer $\omega$ such that $\gamma_{s, u}=0$ for all $s$ and for all $u$ with $|u|>\omega$, and say that the order is $\omega^{*}$ if $\omega^{*}$ is the smallest integer with this property, where we define $\gamma_{s, \emptyset}=1$. Hereafter, we denote the anchored Sobolev space by $H\left(K_{s, \gamma, A}\right)$, and the unanchored Sobolev space by $H\left(K_{s, \gamma, B}\right)$, and let $H\left(K_{s, \gamma}\right)$ be $H\left(K_{s, \gamma, A}\right)$ or $H\left(K_{s, \gamma, B}\right)$.

\subsection{Strong tractability of the Halton sequence}

First, we give the following lemma.

LEMma 4. Let $P_{N}$ be the point set consisting of the first $N$ points of the s-dimensional Halton sequence. Then

$$
e^{2}\left(P_{N} ; H\left(K_{s, \gamma}\right)\right) \leq \frac{1}{N^{2}} \sum_{\emptyset \neq u \subseteq\{1, \ldots, s\}} \gamma_{s, u} \prod_{i \in u}\left(C_{1} i \log _{2} N\right)^{2}
$$

for all $N>1$, where $C_{1}=12 \log 2$.

P r o o f. According to Theorem 16.27 and its proof in [13], we have

$$
e^{2}\left(P_{N} ; H\left(K_{s, \gamma}\right)\right) \leq \sum_{\emptyset \neq u \subseteq\{1, \ldots, s\}} \gamma_{s, u}\left(2^{|u|} D_{|u|}^{*}\left(P_{N}^{u}\right)\right)^{2}
$$

for both anchored and unanchored Sobolev spaces. Applying Corollary 1 completes the proof.

Then, we obtain the main theorem on the tractability using the Halton sequence. Although the proof is almost the same as that of Theorem 16.28 of [13, it is added for the reader's convenience and for completeness. We define $m_{i}=a_{i}^{2}-a_{i}+1 / 3$ for $i=1, \ldots, s$.

Theorem 2. Let $P_{N}$ be the point set of the first $N$ points of the s-dimensional Halton sequence, where $N>1$.

(A) Consider the anchored Sobolev space $H\left(K_{s, \gamma, A}\right)$ with an arbitrary anchor $\mathbf{a}$. 
- For arbitrary finite-order weights $\left\{\gamma_{s, u}\right\}$ of order $\omega$, we have

$$
\frac{e\left(P_{N} ; H\left(K_{s, \gamma, A}\right)\right)}{e\left(0 ; H\left(K_{s, \gamma, A}\right)\right)} \leq \frac{C_{2} s^{\omega} \log _{2}^{\omega} N}{N}
$$

where $C_{2}$ is a positive constant independent of $N$ and $s$.

- If the finite-order weights $\left\{\gamma_{s, u}\right\}$ of order $\omega$ satisfy

$$
M:=\sup _{s \in \mathrm{N}}\left(\frac{\sum_{u \subseteq\{1, \ldots, s\},|u| \leq \omega} \gamma_{s, u} \prod_{i \in u} i^{2}}{\sum_{u \subseteq\{1, \ldots, s\},|u| \leq \omega} \gamma_{s, u} \prod_{i \in u} m_{i}}\right)<\infty,
$$

then for arbitrary $\delta>0$ there exists a positive constant $C_{\delta}$ independent of $N$ and $s$ such that

$$
\frac{e\left(P_{N} ; H\left(K_{s, \gamma, A}\right)\right)}{e\left(0 ; H\left(K_{s, \gamma, A}\right)\right)} \leq C_{\delta} N^{-1+\delta} .
$$

(B) Consider the unanchored Sobolev space $H\left(K_{s, \gamma, B}\right)$.

- For arbitrary bounded finite-order weights $\left\{\gamma_{s, u}\right\}$ of order $\omega$, we have

$$
e\left(P_{N} ; H\left(K_{s, \gamma, B}\right)\right) \leq \frac{C_{3} s^{3 \omega / 2} \log _{2}^{\omega} N}{N},
$$

where $C_{3}$ is a positive constant independent of $N$ and $s$.

- If the finite-order weights $\left\{\gamma_{s, u}\right\}$ of order $\omega$ satisfy

$$
M^{\prime}:=\sup _{s \in \mathrm{N}}\left(\sum_{u \subseteq\{1, \ldots, s\},|u| \leq \omega} \gamma_{s, u} \prod_{i \in u} i^{2}\right)<\infty,
$$

then for arbitrary $\delta>0$ there exists a positive constant $C_{\delta}^{\prime}$ independent of $N$ and $s$ such that

$$
e\left(P_{N} ; H\left(K_{s, \gamma, B}\right)\right) \leq C_{\delta}^{\prime} N^{-1+\delta} .
$$

P r o o f. According to Lemma 4, we have the worst case error as

$$
\begin{aligned}
e^{2}\left(P_{N} ; H\left(K_{s, \gamma, A}\right)\right) & \leq \frac{1}{N^{2}} \sum_{u \subseteq\{1, \ldots, s\}, 0<|u| \leq \omega} \gamma_{s, u} \prod_{i \in u}\left(C_{1} i \log _{2} N\right)^{2} \\
& =\frac{1}{N^{2}} \sum_{\ell=1}^{\omega}\left(\left(C_{1} \log _{2} N\right)^{2 \ell} \sum_{u \subseteq\{1, \ldots, s\},|u|=\ell} \gamma_{s, u} \prod_{i \in u} i^{2}\right) .
\end{aligned}
$$

For the anchored case (A), the initial error is given as

$$
e^{2}\left(0 ; H\left(K_{d, \gamma, A}\right)\right)=\sum_{u \subseteq\{1, \ldots, s\},|u| \leq \omega} \gamma_{s, u} \prod_{i \in u} m_{i} .
$$


First, we consider arbitrary finite-order weights of order $\omega$. Since 1/12 $\leq m_{i} \leq$ $1 / 3$ for all $i=1, \ldots, s$, and from Lemma 4 , we have

$$
\begin{aligned}
\frac{e^{2}\left(P_{N} ; H\left(K_{s, \gamma, A}\right)\right)}{e^{2}\left(0 ; H\left(K_{s, \gamma, A}\right)\right)} & \leq \frac{1}{N^{2}} \frac{\sum_{u \subseteq\{1, \ldots, s\}, 0<|u| \leq \omega} \gamma_{s, u} \prod_{i \in u}\left(C_{1} i \log _{2} N\right)^{2}}{\sum_{u \subseteq\{1, \ldots, s\}, 0 \leq|u| \leq \omega} \gamma_{s, u} \prod_{i \in u} m_{i}} \\
& \leq \frac{12^{\omega}}{N^{2}} \frac{\sum_{u \subseteq\{1, \ldots, s\}, 0<|u| \leq \omega} \gamma_{s, u} \prod_{i \in u}\left(C_{1} i \log _{2} N\right)^{2}}{\sum_{u \subseteq\{1, \ldots, s\}, 0 \leq|u| \leq \omega} \gamma_{s, u}} \\
& \leq \frac{12^{\omega}}{N^{2}} \max _{u:|u| \leq \omega} \prod_{i \in u}\left(C_{1} i \log _{2} N\right)^{2} \\
& \leq \frac{12^{\omega}\left(C_{1} s \log _{2} N\right)^{2 \omega}}{N^{2}}=\frac{C_{2}^{2} s^{2 \omega} \log _{2}^{2 \omega} N}{N^{2}}
\end{aligned}
$$

where $C_{2}=2^{\omega} \sqrt{3^{\omega}} C_{1}^{\omega}$.

Now consider finite-order weights of order $\omega$ satisfying (11). For arbitrary $\delta>0$ define

$$
B_{\delta}=\max _{1 \leq \ell \leq \omega}\left(\left(\frac{C_{1}}{2 \delta \log 2}\right)^{2 \ell}(2 \ell) !\right) .
$$

Then, we have

$$
\begin{aligned}
\frac{e^{2}\left(P_{N} ; H\left(K_{s, \gamma, A}\right)\right)}{e^{2}\left(0 ; H\left(K_{s, \gamma, A}\right)\right)} & \leq \frac{1}{N^{2}} \sum_{\ell=1}^{\omega}\left(\left(C_{1} \log _{2} N\right)^{2 \ell} \frac{\sum_{u \subseteq\{1, \ldots, s\},|u|=\ell} \gamma_{s, u} \prod_{i \in u} i^{2}}{\sum_{u \subseteq\{1, \ldots, s\}, 0 \leq|u| \leq \omega} \gamma_{s, u} \prod_{i \in u} m_{i}}\right) \\
& \leq \frac{M}{N^{2}} \sum_{\ell=1}^{\omega}\left(\frac{C_{1} \log N}{\log 2}\right)^{2 \ell} \leq \frac{B_{\delta} M}{N^{2}} \sum_{\ell=1}^{\omega} \frac{(2 \delta \log N)^{2 \ell}}{(2 \ell) !} \\
& \leq \frac{B_{\delta} M}{N^{2}} \exp (2 \delta \log N)=C_{\delta}^{2} N^{-2+2 \delta},
\end{aligned}
$$

where $C_{\delta}=\sqrt{B_{\delta} M}$. This completes the proof for the case (A).

For the unanchored case (B), in which the initial error $e\left(0 ; H\left(K_{d, \gamma, B}\right)\right)=1$, let $\Gamma^{*}$ satisfy $\gamma_{s, u} \leq \Gamma^{*}$ for all $s$ and for all $u \subseteq\{1, \ldots, s\}$. Then, we have

$$
\begin{aligned}
e^{2}\left(P_{N} ; H\left(K_{s, \gamma, B}\right)\right) & \leq \frac{\Gamma^{*}}{N^{2}} \sum_{\ell=1}^{\omega}\left(\left(C_{1} \log _{2} N\right)^{2 \ell} \sum_{u \subseteq\{1, \ldots, s\},|u|=\ell} \prod_{i \in u} i^{2}\right) \\
& \leq \frac{\Gamma^{*}}{N^{2}} \sum_{\ell=1}^{\omega}\left(s^{2 \ell}\left(C_{1} \log _{2} N\right)^{2 \ell} \sum_{u \subseteq\{1, \ldots, s\},|u|=\ell} 1\right)
\end{aligned}
$$




$$
\begin{aligned}
& \leq \frac{\Gamma^{*} s^{2 \omega}\left(C_{1} \log _{2} N\right)^{2 \omega}}{N^{2}} \sum_{\ell=1}^{\omega}\left(\begin{array}{l}
s \\
\ell
\end{array}\right) \\
& \leq \frac{\Gamma^{*} s^{3 \omega}\left(C_{1} \log _{2} N\right)^{2 \omega}}{N^{2}}=\frac{C_{3}^{2} s^{3 \omega} \log _{2}^{2 \omega} N}{N^{2}},
\end{aligned}
$$

where $C_{3}=\sqrt{\Gamma^{*}} C_{1}^{\omega}$. Note that $\sum_{\ell=1}^{\omega}\left(\begin{array}{l}s \\ \ell\end{array}\right) \leq \sum_{\ell=1}^{\omega} \frac{s^{\ell}}{\ell} \leq s^{\omega}$ for $1 \leq \omega \leq s$.

For the second part of (B), under the condition (12) we have

$$
\begin{aligned}
e^{2}\left(P_{N} ; H\left(K_{s, \gamma, B}\right)\right) & \leq \frac{1}{N^{2}} \sum_{\ell=1}^{\omega}\left(\left(C_{1} \log _{2} N\right)^{2 \ell} \sum_{u \subseteq\{1, \ldots, s\},|u|=\ell} \gamma_{s, u} \prod_{i \in u} i^{2}\right) \\
& \leq \frac{M^{\prime}}{N^{2}} \sum_{\ell=1}^{\omega}\left(\frac{C_{1} \log N}{\log 2}\right)^{2 \ell} \leq C_{\delta}^{\prime 2} N^{-2+2 \delta},
\end{aligned}
$$

where $C_{\delta}^{\prime}=\sqrt{B_{\delta} M^{\prime}}$. Thus, the proof for the case $(\mathrm{B})$ is complete.

\subsection{Strong tractability of the Niederreiter sequence}

We prove the next lemma.

Lemma 5. Let $P_{N}$ be the point set consisting of the first $N$ points of the $s$-dimensional Niederreiter sequence in base $b$. Then

$$
e^{2}\left(P_{N} ; H\left(K_{s, \gamma}\right)\right) \leq \frac{1}{N^{2}} \sum_{\emptyset \neq u \subseteq\{1, \ldots, s\}} \gamma_{s, u} \prod_{i \in u}\left(C_{1} i \log _{2} N\right)^{2}
$$

for all $N>1$, where $C_{1}=\frac{8 b^{2}}{\log _{2} b}>1$ is a constant independent of $N$ and $s$.

Proof. As in the proof of Lemma 4, applying Corollary 2 to (10) completes the proof.

Now we obtain the main theorem on the tractability using the Niederreiter sequence.

Theorem 3. Let $P_{N}$ be the point set of the first $N$ points of the s-dimensional Niederreiter sequence in base $b$, where $N>1$.

(A) Consider the anchored Sobolev space $H\left(K_{s, \gamma, A}\right)$ with an arbitrary anchor a.

- For arbitrary finite-order weights $\left\{\gamma_{s, u}\right\}$ of order $\omega$, we have

$$
\frac{e\left(P_{N} ; H\left(K_{s, \gamma, A}\right)\right)}{e\left(0 ; H\left(K_{s, \gamma, A}\right)\right)} \leq \frac{C_{2} s^{\omega} \log _{2}^{\omega} N}{N},
$$

where $C_{2}$ is a positive constant independent of $N$ and $s$. 
- If the finite-order weights $\left\{\gamma_{s, u}\right\}$ of order $\omega$ satisfy

$$
\sup _{s \in \mathbb{N}}\left(\frac{\sum_{u \subseteq\{1, \ldots, s\},|u| \leq \omega} \gamma_{s, u} \prod_{i \in u} i^{2}}{\sum_{u \subseteq\{1, \ldots, s\},|u| \leq \omega} \gamma_{s, u} \prod_{i \in u} m_{i}}\right)<\infty,
$$

then for arbitrary $\delta>0$ there exists a positive constant $C_{\delta}$ independent of $N$ and $s$ such that

$$
\frac{e\left(P_{N} ; H\left(K_{s, \gamma, A}\right)\right)}{e\left(0 ; H\left(K_{s, \gamma, A}\right)\right)} \leq C_{\delta} N^{-1+\delta} .
$$

(B) Consider the unanchored Sobolev space $H\left(K_{s, \gamma, B}\right)$.

- For arbitrary bounded finite-order weights $\left\{\gamma_{s, u}\right\}$ of order $\omega$, we have

$$
e\left(P_{N} ; H\left(K_{s, \gamma, B}\right)\right) \leq \frac{C_{3} s^{3 \omega / 2} \log _{2}^{\omega} N}{N},
$$

where $C_{3}$ is a positive constant independent of $N$ and $s$.

- If the finite-order weights $\left\{\gamma_{s, u}\right\}$ of order $\omega$ satisfy

$$
\sup _{s \in \mathrm{N}}\left(\sum_{u \subseteq\{1, \ldots, s\},|u| \leq \omega} \gamma_{s, u} \prod_{i \in u} i^{2}\right)<\infty,
$$

then for arbitrary $\delta>0$ there exists a positive constant $C_{\delta}^{\prime}$ independent of $N$ and $s$ such that

$$
e\left(P_{N} ; H\left(K_{s, \gamma, B}\right)\right) \leq C_{\delta}^{\prime} N^{-1+\delta} .
$$

Pro of. Based on Lemma 5 , the proof is done in the same way as that of Theorem 2

\subsection{Strong tractability of the Sobol' sequence}

We show the next lemma.

Lemma 6. Let $P_{N}$ be the point set consisting of the first $N$ points of the s-dimensional Sobol' sequence. Then

$$
e^{2}\left(P_{N} ; H\left(K_{s, \gamma}\right)\right) \leq \frac{1}{N^{2}} \sum_{\emptyset \neq u \subseteq\{1, \ldots, s\}} \gamma_{s, u} \prod_{i \in u}\left(C_{1} i \log _{2} \log _{2}(i+3) \log _{2} N\right)^{2}
$$

for all $N>1$, where $C_{1}>1$ is a constant independent of $N$ and $s$.

Proof. As in the proof of Lemma 4, applying Corollary 3 to (10) completes the proof.

Now we obtain the main theorem on the tractability using the Sobol' sequence. 
TheOREM 4. Let $P_{N}$ be the point set of the first $N$ points of the s-dimensional Sobol' sequence, where $N>1$.

(A) Consider the anchored Sobolev space $H\left(K_{s, \gamma, A}\right)$ with an arbitrary anchor a.

- For arbitrary finite-order weights $\left\{\gamma_{s, u}\right\}$ of order $\omega$, we have

$$
\frac{e\left(P_{N} ; H\left(K_{s, \gamma, A}\right)\right)}{e\left(0 ; H\left(K_{s, \gamma, A}\right)\right)} \leq \frac{C_{2}\left(s \log _{2} \log _{2}(s+3)\right)^{\omega} \log _{2}^{\omega} N}{N},
$$

where $C_{2}$ is a positive constant independent of $N$ and $s$.

- If the finite-order weights $\left\{\gamma_{s, u}\right\}$ of order $\omega$ satisfy

$$
\sup _{s \in \mathrm{N}}\left(\frac{\sum_{u \subseteq\{1, \ldots, s\},|u| \leq \omega} \gamma_{s, u} \prod_{i \in u}\left(i \log _{2} \log _{2}(i+3)\right)^{2}}{\sum_{u \subseteq\{1, \ldots, s\},|u| \leq \omega} \gamma_{s, u} \prod_{i \in u} m_{i}}\right)<\infty,
$$

then for arbitrary $\delta>0$ there exists a positive constant $C_{\delta}$ independent of $N$ and $s$ such that

$$
\frac{e\left(P_{N} ; H\left(K_{s, \gamma, A}\right)\right)}{e\left(0 ; H\left(K_{s, \gamma, A}\right)\right)} \leq C_{\delta} N^{-1+\delta} .
$$

(B) Consider the unanchored Sobolev space $H\left(K_{s, \gamma, B}\right)$.

- For arbitrary bounded finite-order weights $\left\{\gamma_{s, u}\right\}$ of order $\omega$, we have

$$
e\left(P_{N} ; H\left(K_{s, \gamma, B}\right)\right) \leq \frac{C_{3}\left(s^{3 / 2} \log _{2} \log _{2}(s+3)\right)^{\omega} \log _{2}^{\omega} N}{N},
$$

where $C_{3}$ is a positive constant independent of $N$ and $s$.

- If the finite-order weights $\left\{\gamma_{s, u}\right\}$ of order $\omega$ satisfy

$$
\sup _{s \in \mathrm{N}}\left(\sum_{u \subseteq\{1, \ldots, s\},|u| \leq \omega} \gamma_{s, u} \prod_{i \in u}\left(i \log _{2} \log _{2}(i+3)\right)^{2}\right)<\infty,
$$

then for arbitrary $\delta>0$ there exists a positive constant $C_{\delta}^{\prime}$ independent of $N$ and $s$ such that

$$
e\left(P_{N} ; H\left(K_{s, \gamma, B}\right)\right) \leq C_{\delta}^{\prime} N^{-1+\delta} .
$$

Proof. Based on Lemma 6 , the proof is done in the same way as that of Theorem 2 . 


\subsection{Strong tractability of the generalized Faure sequence}

We present the next lemma.

LEMma 7. Let $P_{N}$ be the point set consisting of the first $N$ points of the $s$ -dimensional generalized Faure sequence in base $b$. Then

$$
e^{2}\left(P_{N} ; H\left(K_{s, \gamma}\right)\right) \leq \frac{1}{N^{2}} \sum_{\emptyset \neq u \subseteq\{1, \ldots, s\}} \gamma_{s, u}\left(C_{1} \frac{s \log _{2} N}{\log _{2} s}\right)^{2|u|}
$$

for all $N>1$ and $s>1$, where $C_{1}=8$.

Proof. As in the proof of Lemma 4, applying Corollary 4 to (10) completes the proof.

We now prove the main theorem on the tractability using the generalized Faure sequence.

TheOREM 5. Let $P_{N}$ be the point set of the first $N$ points of the s-dimensional generalized Faure sequence in base b, where $N>1$ and $s>1$.

(A) Consider the anchored Sobolev space $H\left(K_{s, \gamma, A}\right)$ with an arbitrary anchor a.

- For arbitrary finite-order weights $\left\{\gamma_{s, u}\right\}$ of order $\omega$, we have

$$
\frac{e\left(P_{N} ; H\left(K_{s, \gamma, A}\right)\right)}{e\left(0 ; H\left(K_{s, \gamma, A}\right)\right)} \leq \frac{C_{2} s^{\omega} \log _{2}^{\omega} N}{\left(\log _{2}^{\omega} s\right) N}
$$

where $C_{2}$ is a positive constant independent of $N$ and $s$.

- If the finite-order weights $\left\{\gamma_{s, u}\right\}$ of order $\omega$ satisfy

$$
\sup _{s \in \mathrm{N}}\left(\frac{\sum_{u \subseteq\{1, \ldots, s\},|u| \leq \omega} \gamma_{s, u}\left(s / \log _{2} s\right)^{2|u|}}{\sum_{u \subseteq\{1, \ldots, s\},|u| \leq \omega} \gamma_{s, u} \prod_{i \in u} m_{i}}\right)<\infty,
$$

then for arbitrary $\delta>0$ there exists a positive constant $C_{\delta}$ independent of $N$ and $s$ such that

$$
\frac{e\left(P_{N} ; H\left(K_{s, \gamma, A}\right)\right)}{e\left(0 ; H\left(K_{s, \gamma, A}\right)\right)} \leq C_{\delta} N^{-1+\delta} .
$$

(B) Consider the unanchored Sobolev space $H\left(K_{s, \gamma, B}\right)$.

- For arbitrary bounded finite-order weights $\left\{\gamma_{s, u}\right\}$ of order $\omega$, we have

$$
e\left(P_{N} ; H\left(K_{s, \gamma, B}\right)\right) \leq \frac{C_{3} s^{3 \omega / 2} \log _{2}^{\omega} N}{\left(\log _{2}^{\omega} s\right) N},
$$

where $C_{3}$ is a positive constant independent of $N$ and $s$. 
- If the finite-order weights $\left\{\gamma_{s, u}\right\}$ of order $\omega$ satisfy

$$
\sup _{s \in \mathrm{N}}\left(\sum_{u \subseteq\{1, \ldots, s\},|u| \leq \omega} \gamma_{s, u}\left(s / \log _{2} s\right)^{2|u|}\right)<\infty
$$

then for arbitrary $\delta>0$ there exists a positive constant $C_{\delta}^{\prime}$ independent of $N$ and $s$ such that

$$
e\left(P_{N} ; H\left(K_{s, \gamma, B}\right)\right) \leq C_{\delta}^{\prime} N^{-1+\delta} .
$$

Pr o of. Based on Lemma 7 the proof is done in the same way as that of Theorem 2 .

\subsection{Strong tractability of the Xing-Niederreiter sequence and the Hofer-Niederreiter sequence}

We show the next lemma.

Lemma 8. Let $P_{N}$ be the point set consisting of the first $N$ points of the $s$-dimensional Xing-Niederreiter sequence in base $b$ and genus $g$ or of the $s$-dimensional Hofer-Niederreiter sequence in base $b$ and genus $g$. Then

$$
e^{2}\left(P_{N} ; H\left(K_{s, \gamma}\right)\right) \leq \frac{b^{2 g}}{N^{2}} \sum_{\emptyset \neq u \subseteq\{1, \ldots, s\}} \gamma_{s, u} \prod_{i \in u}\left(C_{1} i \log _{2} N\right)^{2}
$$

for all $N>1$, where $C_{1}>1$ is a constant independent of $N$ and $s$.

P r o of. As in the proof of Lemma 4, applying Corollary 5 to (10) completes the proof.

Now we obtain the main theorem on the tractability using the Xing-Niederreiter sequence or the Hofer-Niederreiter sequence.

TheOREM 6. Let $P_{N}$ be the point set of the first $N$ points of the s-dimensional Xing-Niederreiter sequence in base $b$ and genus $g$ or of the $s$-dimensional HoferNiederreiter sequence in base $b$ and genus $g$, where $N>1$.

(A) Consider the anchored Sobolev space $H\left(K_{s, \gamma, A}\right)$ with an arbitrary anchor a.

- For arbitrary finite-order weights $\left\{\gamma_{s, u}\right\}$ of order $\omega$, we have

$$
\frac{e\left(P_{N} ; H\left(K_{s, \gamma, A}\right)\right)}{e\left(0 ; H\left(K_{s, \gamma, A}\right)\right)} \leq \frac{C_{2} s^{\omega} \log _{2}^{\omega} N}{N}
$$

where $C_{2}$ is a positive constant independent of $N$ and $s$. 
- If the finite-order weights $\left\{\gamma_{s, u}\right\}$ of order $\omega$ satisfy

$$
\sup _{s \in \mathrm{N}}\left(\frac{\sum_{u \subseteq\{1, \ldots, s\},|u| \leq \omega} \gamma_{s, u} \prod_{i \in u} i^{2}}{\sum_{u \subseteq\{1, \ldots, s\},|u| \leq \omega} \gamma_{s, u} \prod_{i \in u} m_{i}}\right)<\infty,
$$

then for arbitrary $\delta>0$ there exists a positive constant $C_{\delta}$ independent of $N$ and $s$ such that

$$
\frac{e\left(P_{N} ; H\left(K_{s, \gamma, A}\right)\right)}{e\left(0 ; H\left(K_{s, \gamma, A}\right)\right)} \leq C_{\delta} N^{-1+\delta} .
$$

(B) Consider the unanchored Sobolev space $H\left(K_{s, \gamma, B}\right)$.

- For arbitrary bounded finite-order weights $\left\{\gamma_{s, u}\right\}$ of order $\omega$, we have

$$
e\left(P_{N} ; H\left(K_{s, \gamma, B}\right)\right) \leq \frac{C_{3} s^{3 \omega / 2} \log _{2}^{\omega} N}{N},
$$

where $C_{3}$ is a positive constant independent of $N$ and $s$.

- If the finite-order weights $\left\{\gamma_{s, u}\right\}$ of order $\omega$ satisfy

$$
\sup _{s \in \mathrm{N}}\left(\sum_{u \subseteq\{1, \ldots, s\},|u| \leq \omega} \gamma_{s, u} \prod_{i \in u} i^{2}\right)<\infty,
$$

then for arbitrary $\delta>0$ there exists a positive constant $C_{\delta}^{\prime}$ independent of $N$ and $s$ such that

$$
e\left(P_{N} ; H\left(K_{s, \gamma, B}\right)\right) \leq C_{\delta}^{\prime} N^{-1+\delta} .
$$

Proof. Based on Lemma 8 , the proof is done in the same way as that of Theorem 2.

\section{Discussion}

According to [7, the $s$-dimensional Niederreiter-Xing sequence in base $b$ and genus $g$ [12, in which only rational places are used and thereby global function fields of different genera $g$ are employed for different dimensions $s$, is a $(t, \mathbf{e}, s)$ -sequence in a single base $b$ such that $t=g$ and $e_{1}=\cdots=e_{s}=1$. It is an open problem to determine the tractability using points from the Niederreiter-Xing sequence. Although we know that $t=g=O(s)$, this property makes the problem intractable, because an upper bound for the worst case error must contain a multiplicative factor $b^{O(s)}$, since the $t$-value of any lower dimensional projection $P_{N}^{|u|}$ remains the same, i.e., $t=g$. 


\section{SHU TEZUKA}

Acknowledgements. The first draft of this paper was written during my stay in April 26 through May 1 at the Banach center in Będlewo, where the IBC (Information Based Complexity) 2015 was held. I thank the organizers for inviting me to that beautiful conference. I also thank Anargyros Papageorgiou for his many valuable comments. This research was supported by JSPS KAKENHI(26400202).

\section{REFERENCES}

[1] ATANASSOV, E. I.: On the discrepancy of the Halton sequences, Mathematica Balkanica New Series, 18(1-2) (2004), 15-32.

[2] DICK, J.-PILLICHSHAMMER, F.: Digital Nets and Sequences. Discrepancy Theory and Quasi-Monte Carlo Integration, Cambridge University Press, 2010.

[3] DICK, J.-NIEDERREITER, H.-PILLICHSHAMMER, F.: Weighted star discrepancy of digital nets in prime bases. In: Monte Carlo and quasi-Monte Carlo methods 2004, Springer, (2006), 77-96.

[4] DUSART, P.: The $k^{\text {th }}$ prime is greater than $k(\ln k+\ln \ln k-1)$ for $k \geq 2$, Mathematics of Computation, 68 (1999), 411-415.

[5] HALTON, J. H.: On the efficiency of certain quasi-random sequences of points in evaluating multi-dimensional integrals, Numerische Mathematik, 2 (1960), 84-90.

[6] HICKERNELL, F. J.-WANG, X.: The error bounds and tractability of quasi-Monte Carlo algorithms in infinite dimension, Mathematics of Computation, 71 (2002), 1641-1661.

[7] HOFER, R.-NIEDERREITER, H.: A construction of $(t, s)$-sequences with finite-row generating matrices using global function fields, Finite Fields and Their Applications, 21 (2013), 97-110.

[8] JÄCKEL, P.: Monte Carlo Methods in Finance, John Wiley and Sons, 2002.

[9] KRUSE, M.-STICHTENOTH, H.: Ein Analogon zum Primzahlsatz für algebraische Funktionenkörper, Manuscripta Mathematica, 69 (1990), 219-221.

[10] MATOUŠEK, J.: Geometric Discrepancy: An Illustrated Guide, revised second printing, Springer, 2010.

[11] NIEDERREITER, H.: Random Number Generation and Quasi-Monte Carlo Methods, In: CBMS-NSF Regional Conference Series in Applied Mathematics. Vol. 63, SIAM, 1992.

[12] NIEDERREITER, H.-XING, C.: Low-discrepancy sequences and global function fields with many rational places, Finite Fields and Their Applications, 2 (1996), 241-273.

[13] NOVAK, E.-WOŹNIAKOWSKI, H.: Tractability of Multivariate Problems, Volume II: Standard Information for Functionals, EMS Tracts in Mathematics Vol. 12. European Mathematical Society (EMS), Zrich, 2010.

[14] PAPAGEORGiOU, A.-TRAUB, J. F.: Beating Monte Carlo, RISK, 9 (June 1996), 63-65.

[15] SLOAN, I. H.-WOŹNIAKOWSKI, H.: When are quasi-Monte Carlo algorithms efficient for high dimensional integrals, Journal of Complexity, 14 (1998), 1-33.

[16] SLOAN, I. H.-WANG, X.-WOŹNIAKOWSKI, H.: Finite-order weights imply tractability of multivariate integration, Journal of Complexity, 20 (2004), 46-74. 


\section{TRACTABILITY OF MULTIVARIATE INTEGRATION}

[17] SOBOL, I. M.: The distribution of points in a cube and the approximate evaluation of integrals, USSR Computational Mathematics and Mathematical Physics, 7 (1967), 86-112.

[18] TEZUKA, S.: Uniform Random Numbers: Theory and Practice, The Kluwer International Series in Engineering and Computer Science Vol. 315. Kluwer Academic Publishers, Dordrecht, 1995.

[19] Financial applications of Monte Carlo and quasi-Monte Carlo methods, (P. Hellekalek and G. Larcher, eds.) In: Random and Quasi-Random Point Sets. Lecture Notes in Statistics, 138, Springer, (1998), 303-332.

[20] On the discrepancy of generalized Niederreiter sequences, Journal of Complexity, 29 (2013), 240-247.

[21] (t form Distribution Theory, Ostravice, (June, 2014).

[22] _ Improvement on the discrepancy of $(t, \mathbf{e}, s)$-sequences, Tatra Mountains Mathematical Publications, 59 (2014), 27-38.

[23] TRAUB, J. F.-WERSCHUlZ, A. G.: Complexity and Information, (Lezioni Lincee). Cambridge University Press, 1998.

[24] TRAUB, J. F.-WOŹNIAKOWSKI, H.: Breaking intractability, Scientific American, (January 1994), 102-107.

[25] WANG, X.: A constructive approach to strong tractability using quasi-Monte Carlo algorithms, Journal of Complexity, 18 (2002), 683-701.

[26] Strong tractability of multivariate integration using quasi-Monte Carlo algorithms, Mathematics of Computation, 72 (2003), 823-838.

[27] WOŹNIAKOWSKI, H.: Average case complexity of multivariate integration, Bulletin of the American Mathematical Society, 24 (1991), 185-194.

[28] Tractability of multivariate problems, Foundations of Computational Mathematics, Hong Kong 2008, London Math. Soc. Lecture Note Ser. Vol. 363, Cambridge University Press, Cambridge 2009, 236-276.

[29] XING, C.-NIEDERREITER, H.: A construction of low-discrepancy sequences using global function fields, Acta Arithmetica, 73 (1995), 87-102.

Received November 23, 2015

Accepted April 30, 2016

\section{Shu Tezuka}

Institute of Mathematics for Industry, Kyushu University,

744 Motooka, Nishi-ku, Fukuoka-shi, Fukuoka-ken, JAPAN 819-0395

E-mail: tezuka@imi.kyushu-u.ac.jp 